\title{
Early detection of SARS-CoV-2 in circulating immune cells in a mouse model
}

$4{ }^{1}$ Department of Immunology, School of Medicine, University of Connecticut Health Center,

5 Farmington, CT 06030, USA

$6{ }^{2}$ QCDx LLC, 400 Farmington Ave, Farmington, CT 06032, USA.

7

$8 \quad$ * T.G. and S.K. contributed equally

9

10 Address correspondence to:

11 Penghua Wang, Ph.D., Department of Immunology, School of Medicine, University of

12 Connecticut Health Center, Farmington, CT 06030, USA. Email: pewang@uchc.edu, Tel: 860-

$13 \quad 679-6393$.

14 Triantafyllos Tafas Ph.D., QCDx LLC, 400 Farmington Ave, Farmington, CT 06032. Email:

15 fyl.tafas@qcd-x.com, Tel: 860-679-4673.

16

17 Word count for the abstract: 98

18 Word count for the main text: 1992 


\section{Footnote}

21

22 Conflict of Interest: T.G. and P.W. declare no competing financial interest. S.K. and T.T. are

23 employed by QCDx LLC.

24

25 Funding Source: National Institutes of Health grant R01Al132526 to P.W.

University of Connecticut Health Center grant G401894 to P.W.

28 Previous presentation: None

29

30 Corresponding Author Contact Information: Penghua Wang, Ph.D., 263 Farmington Avenue,

31 Farmington, CT 06030; Tel: 860-679-6393; Email: pewang@uchc.edu 


\section{ABSTRACT}

33 SARS-CoV-2 infects the respiratory tract, lung and then other organs. However, its

34 pathogenesis remains largely unknown. We used RareScope ${ }^{\mathrm{TM}}$ Fluorescence Light Sheet

35 Microscopy (FLSM) and fluorescent in situ hybridization of RNA (RNA-FISH) to detect SARS-

36 CoV-2 RNA and dissemination kinetics in mouse blood circulation. By RNA-FISH, we found

37 that SARS-CoV-2 RNA-positive leukocytes, including CD11c cells, appeared as early as one

38 day after infection and continued through day 10 post infection. Our data suggest that SARS-

39 CoV-2-permissive leukocytes contribute to systemic viral dissemination, and RNA-FISH

40 combined with FLSM can be utilized as a sensitive tool for SARS-CoV-2 detection in blood

41 specimens.

42

43 Key words: SARS-CoV-2, viral, pathogenesis, RNA-FISH, FLSM, detection 


\section{Background}

45 The (+) single-stranded (ss) RNA coronaviruses (CoV) are major cause of fatal human

46 respiratory diseases, such as Severe Acute Respiratory Syndrome (SARS)-causing CoV and

47 Middle East Respiratory Syndrome (MERS)-CoV. SARS started in November 2002 in Southern

48 China, spread to 26 countries, and resulted in 8439 cases and 821 deaths[1]. Between its

49 discovery in 2012 and January 2020, MERS-CoV caused 2519 cases and 866 deaths[2]. At the

50 end of 2019, a new SARS strain, SARS-CoV-2, which is $86 \%$ identical to SARS-CoV-1 at the

51 amino residue level, emerged in humans in Central China and now has spread worldwide. As of

52 today, there are $>34$ million confirmed SARS-CoV-2 cases and $>1$ million deaths from $\sim 250$

53 countries[3], constituting the greatest global public health crisis in the $21^{\text {st }}$ century.

54 Once a human SARS-CoV gains entry through the respiratory tract, airway epithelial

55 cells, alveolar epithelial cells, vascular endothelial cells and alveolar macrophages are among

56 the first target $[4,5]$. These cell types are suspected to be 'ground-zero' for early infection and

57 subsequent replication [6, 7]. In particular, proinflammatory monocyte-derived macrophages

58 were dominant in the bronchoalveolar lavage fluid from patients with severe COVID-19 [8].

59 These SARS-CoV-2 permissive cells could contribute to lung inflammation and viral

60 dissemination to other organs.

61 We here employed a mouse model and RareScope ${ }^{\mathrm{TM}}$ Fluorescence Light Sheet Microscopy

62 (FLSM) to examine early dissemination of SARS-CoV-2 through the blood circulation. We report

63 that SARS-CoV-2 positive leukocytes appear early after infection, which could help this virus

64 spread systemically. 


\section{Methods}

\section{Cell and virus culture}

Vero cells (monkey kidney epithelial cells, Cat. \# CCL-81) were purchased from ATCC (Manassas, VA, USA). The cells were grown at $37^{\circ} \mathrm{C}$ and $5 \% \mathrm{CO}_{2}$ in complete DMEM medium:

69 Dulbecco's modified Eagle medium (DMEM) (Corning) supplemented with 10\% fetal bovine 70 serum (FBS) (Gibco) and 1\% penicillin-streptomycin (P/S; Corning). These cell lines are not

71 listed in the database of commonly misidentified cell lines maintained by ICLAC, and in our

72 hands tested negative for mycoplasma contamination. In order to ensure cell cultures are 73 mycoplasma free, we regularly treated cells with MycoZap (Lonza). SARS-CoV-2 (NR-52281

74 SARS-related coronavirus 2, isolate USA-WA1/2020) was propagated in Vero cells and 75 concentrated with a polyethylene glycol (PEG) (Cat\# LV-810A, System Biosciences, Palo Alto, 76 CA 94303, USA) to a titer of $\sim 1 \times 10^{7}$ plaque forming units (PFU)/ml. Adeno 5 virus expressing

77 the receptor for SARS-CoV-2, human Angiotensin Converting Enzyme 2 (hACE2) was custommade by VectorBuilder Inc. (Chicago, IL 60609, USA).

Mouse infection and sample collection

Mouse experiments were approved and performed according to the guidelines of

82 the Institutional Animal Care and Use Committee at Yale University. 8-10 weeks-old female

83 C57BL/6J mice (JAX Stock \#: 000664) were inoculated with 2x10 PFU of Ad5-hACE2 by

84 intranasal instillation. Five days after Ad5 transduction, three mice were subsequentially infected

85 with $2 \times 10^{5}$ PFU of SARS-CoV-2 through the intranasal route in the BSL-3 facility at Yale

86 University, New Haven, CT. Whole blood was collected retro-orbitally at different time point after

87 anesthesia using $30 \% \mathrm{v} / \mathrm{v}$ isoflurane diluted in propylene glycol. Approximately $100 \mu \mathrm{l}$ whole

88 blood were collected for RNA-FISH and $50 \mu$ for RNA isolation and Quantitative PCR (qPCR).

89 For tissue collection, mice were euthanized in $100 \%$ Isoflurane. About $20 \mathrm{mg}$ of left lung tissue

90 was harvested at the indicated time point for western, and $20 \mathrm{mg}$ of left lung tissue for RNA 
91 isolation and qPCR. Day 0 mouse samples were taken from uninfected animals collected and

92 isolated in the same manner with Ad5 transduction.

93

94 RNA-FISH and RareScope ${ }^{\text {TM }}$ Fluorescence Light Sheet Microscopy

95 An RNA FISH probe was developed against the SARS-CoV-2 Spike gene. The probe

96 was designed based on the published SARS-CoV-2 genome

97 (https://www.ncbi.nlm.nih.gov/nuccore/MN985325) and consists of 48 individual oligomer-

98 primers, custom-built by Cambridge Bioscence (Cambridge, CB23 8SQ, United Kingdom)

99 (Supplementary Table 1). The full Spike RNA target sequence is 1,673 nt in length and the 100 chosen oligomers cover independent 20nt sequences. The 48 oligomers underwent basic local

101 alignment search tool (BLAST) analysis to eliminate off-target hybridization. Each oligomer has

102 a fluorescent tail of Quasar 670 (LGC Biosearch Technologies, Middlesex, UK). All white blood

103 cells (WBCs) from each mouse and each time-point blood samples were fluorescently

104 immunostained in solution. Signals for 5 fluorescent markers were created by staining with

105 antibodies against the common leukocyte antigen (Rat-anti-mouse CD45, Cat\# 550539, clone:

106 30-F11, BD Pharmingen, San Jose, CA 95131, USA) indirectly labeled with a goat-anti-rat

107 secondary antibody labeled with Alexa Fluor 488, a dendritic cell anti-CD11c marker (Hamster-

108 anti-Mouse CD11c, Cat\# 553799, clone HL3, BD Pharmingen) indirectly labeled with goat-anti-

109 Hamster Alexa Fluor 594, hACE2 (Mouse-anti-Human ACE-2, Cat\# sc-390851, clone E-11,

110 Santa Cruz Biotechnology) indirectly labeled with goat-anti-mouse Alexa Fluor 594, and the

111 RNA-FISH Spike Probe fluorescently labeled with Quasar 670. Nuclei were counterstained by

112 Hoechst 33342.

113 After staining, the morphologically intact cells were immobilized in hydrogel into

114 RarePrep ${ }^{\mathrm{TM}}$ specimen fixtures that were loaded in the RareScope 4D (X, Y, Z and rotational)

115 microscope stage for 3-dimensional (3-D) imaging. The RarePrep fixture presents the cylindrical,

116 transparent suspension of immobilized cells to the RareScope FLSM optical path where it is 
117 scanned in an automated fashion. Utilizing proprietary script programs created on the

118 Fiji/lmageJ software platform, 3-D image stacks of the immobilized cells are acquired,

119 individually for each of the 5 fluorescent markers. The 3-D image stacks from each blood

120 sample were analyzed by expert reviewers and more than five hundred WBCs counted to verify

121 presence of SARS-CoV-2 signals, totaling 1500-2000 cells from each mouse and each time-

122 points.

123

124 RNA extraction and Quantitative reverse-transcription PCR

125 Total RNA was isolated from whole blood and lung tissue using a PureLink RNA Mini kit

126 (Invitrogen, Germantown, MD 20874, USA). All the blood and tissue samples were kept in RNA

127 Lysis Buffer in $-80 \%$ before RNA purification. Reverse transcription was performed using a

128 PrimeScript $^{\mathrm{TM}}$ RT Reagent Kit (Takara Bio, Mountain View, CA 94043 USA). qPCR was

129 performed with gene specific primers and SYBR Green (iTaq Universal SYBR Green Supermix,

130 Bio-Rad, Hercules, CA 94547, USA). The primers for SARS-CoV-2 were published by the

131 Centers for Disease Control and Prevention of United States of America: forward primer (5'-

132 GAC CCC AAA ATC AGC GAA AT -3') and reverse primer (5'- TCT GGT TAC TGC CAG TTG

133 AAT CTG -3'). The primers for hACE2 were forward primer (5'-

134 ATCTGAGGTCGGCAAGCAGC-3)' and reverse primer (5'-CAATAATCCCCATAGTCCTC-3').

135 The primers for the housekeeping gene control mouse beta actin, Actb, where: forward primer

136 (5'- AGAGGGAAATCGTGCGTGAC -3') and reverse primer (5'-

137 CAATAGTGATGATGACCTGGCCGT-3'). The following PCR cycling program was used: 10 min 138 at $95^{\circ}$, and 40 cycles of $15 \mathrm{sec}$ at $95^{\circ}$ and $1 \mathrm{~min}$ at $60^{\circ} \mathrm{C}$. 


\section{RESULTS}

SARS-CoV-2 is transmitted primarily through respiratory droplets, infects the respiratory

143 tract, lung and then disseminates to other organs likely through the blood circulation. However,

144 its dissemination kinetics are unknown. To this end, we tested if SARS-CoV-2 positive white

145 blood cells could be detected using a mouse model. Generally speaking, human SARS-CoV-2

146 does not infect efficiently or cause overt disease in mice [9]. However, human ACE2 (hACE2, a

147 major cellular entry receptor for SARS-CoV-2)-transgenic [9] or transiently transduced mice are

148 susceptible to SARS-CoV-2 infection and develop lung pathology [10]. Thus, we transiently

149 expressed human ACE2 in mice using a non-replicating adenovirus 5 vector and then infected

150 them with SARS-CoV-2. hACE2 expression and SARS-CoV-2 are largely restricted to mouse

151 lungs [10]. In our Ad5-hACE2 mouse model, hACE2 was successfully expressed in lung tissue

152 (Fig. 1A), and SARS-CoV-2 was positive in lung after 4 days of infection (Fig. 1B). As a control,

153 we first validated the SARS-CoV-2-RNA probe in heavily infected Vero cells (Fig.1C). Then, we

154 included a human blood sample (without SARS-CoV-2 infection) as control in which hACE2 was

155 expressed at a high level. SARS-CoV-2 + / CD45+ cells were detected in mice two days post

156 infection (Fig.1D).

157 After validation of the mouse model and RNA-FISH probe, a cohort of 3 mice were

158 tested longitudinally over a period of 8 days. Blood sample of $100 \mu$ l each mouse was collected

159 retro-orbitally before SARS-CoV-2 infection (0 DPI) and none of the three blood samples had

160 SARS-CoV-2 ${ }^{+}$Spike mRNA signatures in WBC (Fig. 2A, 2B). The same three mice were

161 infected with SARS-CoV-2, and blood was collected on 1, 3, and 8 DPI. On the first day post

162 infection (1 DPI), we observed that leukocytes were positive for SARS-CoV-2 Spike mRNA

163 (1.08\%), and SARS-CoV- $2^{+}+$cells increased slightly in frequency to a peak of about $1.27 \%$ on 3

164 DPI and then declined to about $0.28 \%$ on 8 DPI (Fig. 2A, 2B). There was a statistically

165 significant difference between groups, based on Single Factor ANOVA $(F(4,12)=17.39$,

$166 \mathrm{p}=.0007)$ comparing the change in mean frequency of spike positive cells overtime. The Spike 
167 mRNA signal was robust with diverse morphology ranging from well-defined FISH dots to

168 disperse clouds of SARS-CoV-2 signal positivity in CD45 and CD11c positive cells (Fig. 2C).

169 Another $50 \mu$ l of whole blood was collected at the same time in the same cohort for RNA

170 extraction and quantitative RT-PCR (qPCR) detection of SARS-CoV-2 RNA. No blood

171 specimens tested positive for SARS-CoV-2 by qPCR with a cut off threshold cycle (Ct) set at 40

172 (Supplementary Table 2).

173

174 DISCUSSION

175 Once the virus gains entry through the respiratory tract, the first cells to be infected are 176 airway epithelial cells, alveolar epithelial cells, vascular endothelial cells and alveolar 177 macrophages [4, 5]. SARS-CoV-2 may also infect immature and mature human monocyte178 derived DCs [11]. Subsequently, viruses disseminate through the blood circulation to other 179 permissive organs. We here demonstrated the presence of SARS-CoV-2 Spike mRNA-positive

180 WBCs by RNA-FISH rapidly, at day 1 post infection (Fig.2C). Interestingly, a large portion of the 181 SARS-CoV-2-carrying WBCs were innate CD11c-positive cells on 1 DPI. CD11c is most 182 prominently expressed by dendritic cells, but also by monocytes, macrophages, neutrophils, and 183 some B cells. Since in our mouse model SARS-CoV-2 infection is limited to the lung [10], these 184 circulating SARS-CoV-2-positive immune cells are likely to have originated from immune 185 infiltrates and/or resident immune cells in the lung. These cells could have been rapidly 186 activated to produce innate antiviral immune responses, and to activate adaptive immunity, 187 including dendritic cell trafficking to lymph nodes early in infection. Indeed, viral infection triggers 188 rapid differentiation of human blood monocytes into dendritic cells (DCs) with enhanced 189 capacity to activate T cells [12]. However, circulating SARS-CoV-2-positive DCs could help virus 190 spread to other tissues. This has been observed in dengue virus infection, which depends on

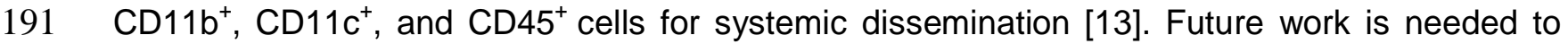
192 include more immunocyte markers to identify the different categories of WBC which contribute 
193 to SARS-CoV-2 dissemination. We recognize that expression of hACE2 by an adenovirus

194 vector may potentially alter the cell tropism of SARS-CoV-2 because of a broad cell tropism of

195 adenoviruses. Nonetheless, our results still provide insight into early responsive immune cells

196 and viral dissemination.

197 Another intriguing finding of this study is sensitive detection of SARS-CoV-2 by RNA-

198 FISH coupled with RareScope microscopy. In our experimental conditions, SARS-CoV-2 RNA

199 was undetectable in the blood throughout all time points. RNA-FISH coupled with RareScope

200 microscopy method seems more sensitive for detection of SARS-CoV-2 in blood than RT-PCR.

201 The enhanced sensitivity of RNA-FISH-based detection is likely because, with

202 RareScope-based detection, the cell morphology and viral RNA integrity are preserved in the

203 cell suspension immobilized in a hydrogel. Furthermore, cumulative signals from 48 individual

20420 nt-oligomers in the SARS-CoV-2 probe targeting the viral Spike gene may increase

205 sensitivity. A potential concern about RNA-FISH could be future mismatch between probes and

206 the SARS-CoV-2 genome due to rapid mutation [14], which could be also a problem to qPCR

207 with two primers. However, this problem can be overcome by timely sequencing of new clinical

208 SARS-CoV-2 isolates. By far, the Spike gene is very well conserved among the clinical isolates

209 worldwide [15]. Of note, even with a 48-oligomer probe, the RNA-FISH method demonstrated

210 high specificity as all uninfected blood samples were negative for SARS-CoV-2 RNA. Therefore,

211 our RNA-FISH method is likely a more robust diagnostic with blood specimens than qPCR.

212 Future work is to test our method with clinical blood specimens of COVID-19 patients. 
bioRxiv preprint doi: https://doi org/10.1101/2021.06.30.450531; this version posted June 30, 2021. The copyright holder for this preprint (which was not certified by peer review) is the author/funder, who has granted bioRxiv a license to display the preprint in perpetuity. It is made available under aCC-BY-NC-ND 4.0 International license.

\section{Author contributions}

215 T.G. performed the animal and qPCR work; S.K. performed the RNA-FISH and microscopy. T.T.

216 and P.W. conceived and supervised the study. T.G., S.K., T.T. and P.W. wrote the manuscript.

217 All the authors reviewed and/or modified the manuscript.

218 We are thankful to Anthony T. Vella, Ph.D. for helping with textual editing. 


\section{REFERENCES}

220 1. WHO. Cumulative Number of Reported Probable Cases of SARS.

221 https://wwwwhoint/csr/sars/country/2003_07_04/en/ 2003.

222 2. WHO. Middle East respiratory syndrome coronavirus (MERS-CoV).

223 https://wwwwhoint/emergencies/mers-cov/en/ 2020.

224 3. WHO. Coronavirus disease (COVID-2019) situation reports.

225 https://wwwwhoint/emergencies/diseases/novel-coronavirus-2019/situation-reports/ 2020.

226 4. Jia HP, Look DC, Shi L, et al. ACE2 Receptor Expression and Severe Acute Respiratory

227 Syndrome Coronavirus Infection Depend on Differentiation of Human Airway Epithelia. Journal 228 of Virology 2005; 79:14614-21.

229 5. Hamming I, Timens W, Bulthuis M, Lely A, Navis G, Goor Hv. Tissue distribution of ACE2 230 protein, the functional receptor for SARS coronavirus. A first step in understanding SARS 231 pathogenesis. The Journal of Pathology 2004; 203:631-7.

232 6. Ziegler CGK, Allon SJ, Nyquist SK, et al. SARS-CoV-2 receptor ACE2 is an interferon233 stimulated gene in human airway epithelial cells and is detected in specific cell subsets across 234 tissues. Cell 2020.

235 7. Li MY, Li L, Zhang Y, Wang XS. Expression of the SARS-CoV-2 cell receptor gene ACE2 in 236 a wide variety of human tissues. Infectious Diseases of Poverty 2020; 9.

237 8. Liao M, Liu Y, Yuan J, et al. Single-cell landscape of bronchoalveolar immune cells in 238 patients with COVID-19. Nature Medicine 2020.

239 9. Bao L, Deng W, Huang B, et al. The Pathogenicity of SARS-CoV-2 in hACE2 Transgenic 240 Mice. bioRxiv 2020:2020.02.07.939389.

241 10. Sun J, Zhuang Z, Zheng J, et al. Generation of a Broadly Useful Model for COVID-19

242 Pathogenesis, Vaccination, and Treatment. Cell 2020; 182:734-43 e5. 
243 11. Yilla M, Harcourt BH, Hickman CJ, et al. SARS-coronavirus replication in human peripheral

244 monocytes/macrophages. Virus Res 2005; 107:93-101.

245 12. Hou W, Gibbs JS, Lu X, et al. Viral infection triggers rapid differentiation of human blood

246 monocytes into dendritic cells. Blood 2012; 119:3128-31.

247 13. Pham AM, Langlois RA, TenOever BR. Replication in cells of hematopoietic origin is

248 necessary for Dengue virus dissemination. PLoS Pathog 2012; 8:e1002465.

249 14. Shen Z, Xiao Y, Kang L, et al. Genomic Diversity of Severe Acute Respiratory Syndrome-

250 Coronavirus 2 in Patients With Coronavirus Disease 2019. Clin Infect Dis 2020; 71:713-20.

251 15. Pachetti M, Marini B, Benedetti F, et al. Emerging SARS-CoV-2 mutation hot spots include

252 a novel RNA-dependent-RNA polymerase variant. J Transl Med 2020; 18:179. 


\section{FIGURE LEGENDS}

\section{Figure 1 Validation of the Ad5-hACE2 mouse model and RNA-FISH probe.}

256 A) Western blots of lung tissue from Ad5-hACE2 mouse and Ad5-Vec control mouse. B) qPCR 257 quantification of SARS-CoV2 virus loads in lung tissue of Ad5-hACE2 mouse before and 4 days 258 after SARS-CoV2 infection. C) Images of Vero cells as positive and negative experimental 259 controls. Mock and infected Vero cells were stained with SARS-CoV2 RNA FISH and an 260 epithelial immunofluorescent (IF) marker [pan-Cytokeratin (Pan-CK) specific for 18 different 261 clones of Cytokeratin]. Vero composite images use blue for nucleus, green for pan-CK, and red 262 for CoV-2 Spike RNA. D) Immunofluorescent images of white blood cells (WBC) from human 263 and mouse. WBCs were fixed and stained for CD45 indirectly labeled with Alexa Fluor 488, 264 human ACE2 indirectly labeled with Alexa Fluor 594 and the RNA-FISH Spike probe directly 265 conjugated with Quasar 670. Nuclear DNA was counterstained by Hoechst 33342 . The 266 composite images are the combination of nuclear DNA (blue), CD45 (green), hACE2(yellow) 267 and SAR-CoV-2 Spike RNA (Red). The images were acquired with a RareScope FLSM 268 microscope with a water immersion 20X, NA 0.5 objective lens.

270 Figure 2 Detection of SARS-CoV2 RNA in white blood cells by RNA-FISH.

271 A) The percentage of SARS-CoV2-positive WBC. Bars: mean \pm s.e.m. Each $(\bullet)$ symbol $=$ one

272 animal. B) A summary of threshold cycle (Ct) of qPCR of SARS-CoV-2 RNA at different days 273 post infection (DPI). Each row indicates one mouse blood sample with technical triplicate. Actb

274 is a mouse house-keeping gene. N.A (not applicable): Ct greater than 40. C)

275 Immunofluorescent images of white blood cells (WBC) from different days post infection (DPI).

276 WBCs were fixed and stained for CD45 indirectly labeled with Alexa Fluor 488, goat-anti-

277 hamster CD11c indirectly labeled with Alexa Fluor 594 and the RNA-FISH Spike probe directly

278 conjugated with Quasar 670. Nuclear DNA was counterstained by Hoechst 33342 . The 279 composite images are the combination of nuclear DNA (blue), CD45 (green), CD11c(yellow) 
bioRxiv preprint doi: https://doi.org/10.1101/2021.06.30.450531; this version posted June 30, 2021. The copyright holder for this preprint (which was not certified by peer review) is the author/funder, who has granted bioRxiv a license to display the preprint in perpetuity. It is made available under aCC-BY-NC-ND 4.0 International license.

280 and SAR-CoV-2 Spike RNA (Red). The images were acquired with a RareScope FLSM

281 microscope with a water immersion 20X, NA 0.5 objective lens. 
bioRxiv preprint doi: https://doi org/10.1101/2021.06.30.450531; this version posted June 30, 2021. The copyright holder for this preprint (which was not certified by peer review) is the author/funder, who has granted bioRxiv a license to display the preprint in perpetuity. It is made available under aCC-BY-NC-ND 4.0 International license.

\section{Supplementary data}

283

284 Table 1 The list of Quasar 670 Fluorescently labelled primers included in the RNA-FISH probe

285 for the SARS-CoV2 Spike gene.

286

287 Table 2 The total counts of SARS-CoV-2 Spike RNA positive cell in WBCs with the percent of 288 Spike RNA positive cells out of total nuclei. 
Fig.1

A

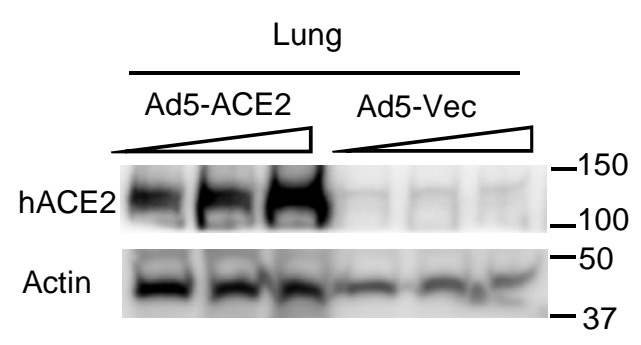

C

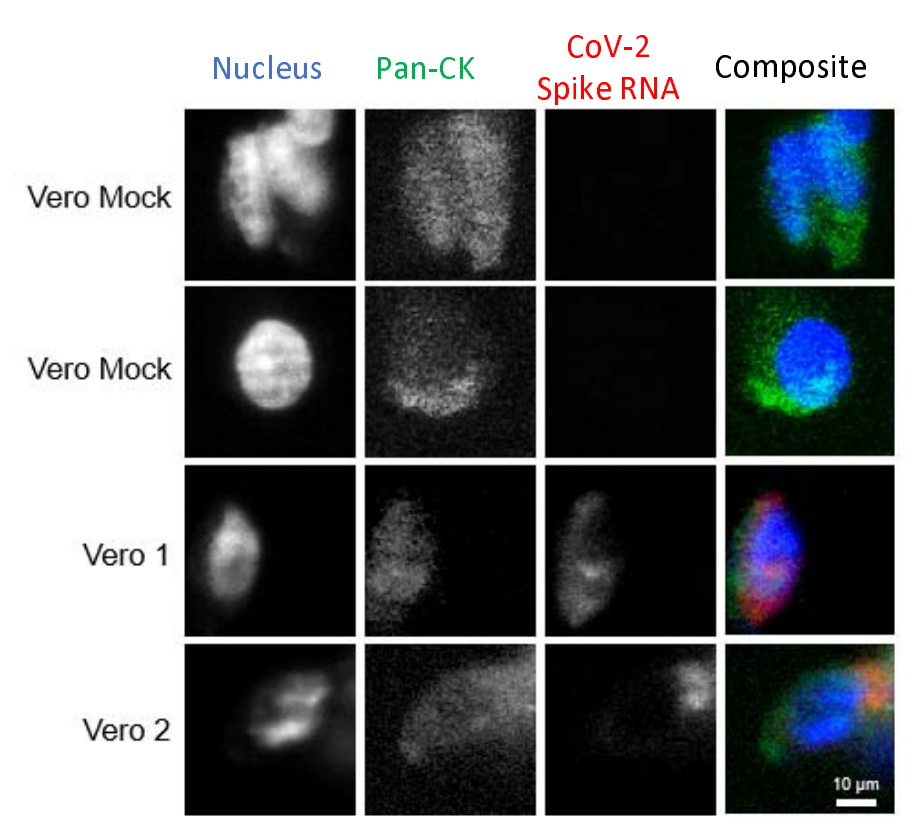

B

\begin{tabular}{|c|c|c|c|c|}
\hline & \multicolumn{2}{|c|}{ Actb } & \multicolumn{2}{c|}{ SARS-CoV-2 } \\
\hline Lung Day0 & 18.91 & 18.94 & NA & NA \\
\hline Lung Day4 & 19.96 & 20.01 & 18.99 & 19.04 \\
\hline
\end{tabular}

D

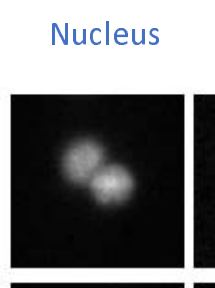

CD45

hACE-2

CoV-2 Composite

Human
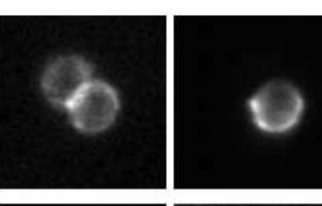
Spike RNA

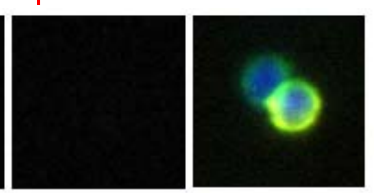

Mouse

Mock

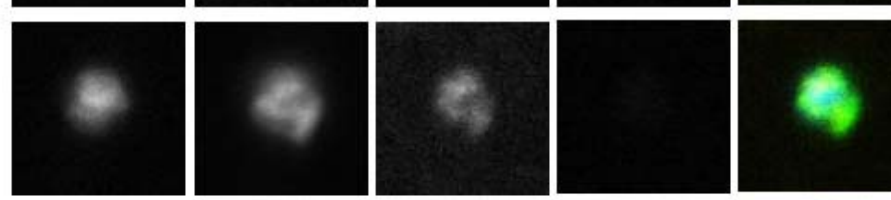

Mouse

2 DPI
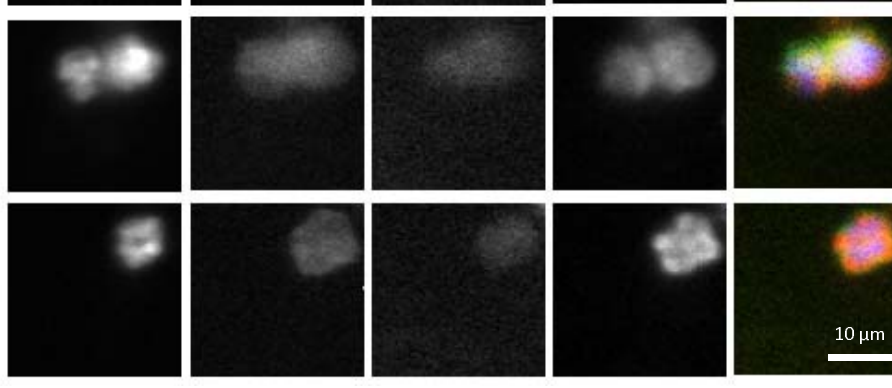

Mouse

$2 \mathrm{DPI}$ 
Fig.2

A

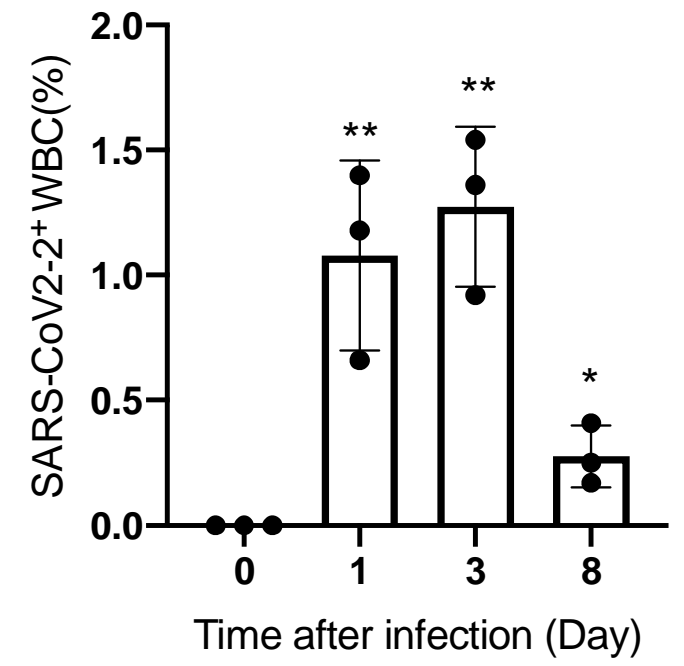

C

\begin{tabular}{|c|c|c|c|c|c|}
\hline & $\begin{array}{c}\text { Sample } \\
\text { ID }\end{array}$ & $\begin{array}{l}\text { Spike } \\
\text { RNA + }\end{array}$ & Nuclei & Ratio & Averages \\
\hline \multirow{3}{*}{ Day0 } & M1 & 0 & 2571 & $0.00 \%$ & \multirow{3}{*}{$0.00 \%$} \\
\hline & $\mathrm{M} 2$ & 0 & 1000 & $0.00 \%$ & \\
\hline & M3 & 0 & 1000 & $0.00 \%$ & \\
\hline \multirow{3}{*}{ Day1 } & M1 & 32 & 2710 & $1.18 \%$ & \multirow{3}{*}{$1.08 \%$} \\
\hline & M2 & 22 & 3357 & $0.66 \%$ & \\
\hline & M3 & 32 & 2292 & $1.40 \%$ & \\
\hline \multirow{3}{*}{ Day3 } & M1 & 24 & 2606 & $0.92 \%$ & \multirow{3}{*}{$1.27 \%$} \\
\hline & M2 & 28 & 1822 & $1.54 \%$ & \\
\hline & M3 & 56 & 4117 & $1.36 \%$ & \\
\hline \multirow{3}{*}{ Day8 } & M1 & 15 & 6114 & $0.25 \%$ & \multirow{3}{*}{$0.28 \%$} \\
\hline & $\mathrm{M} 2$ & 10 & 2450 & $0.41 \%$ & \\
\hline & M3 & 2 & 1183 & $0.17 \%$ & \\
\hline
\end{tabular}

B Nucleus

D0

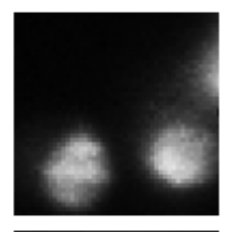

D0

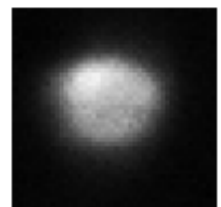

D1

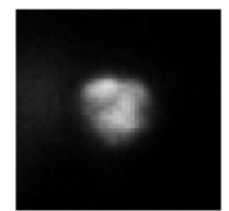

D1

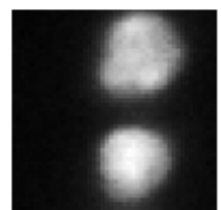

D3

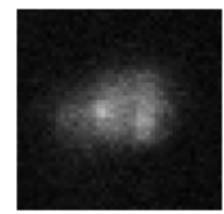

D3

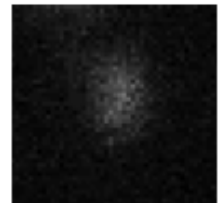

D8

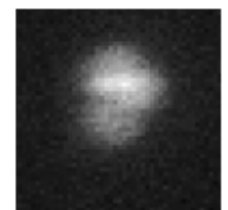

CD45
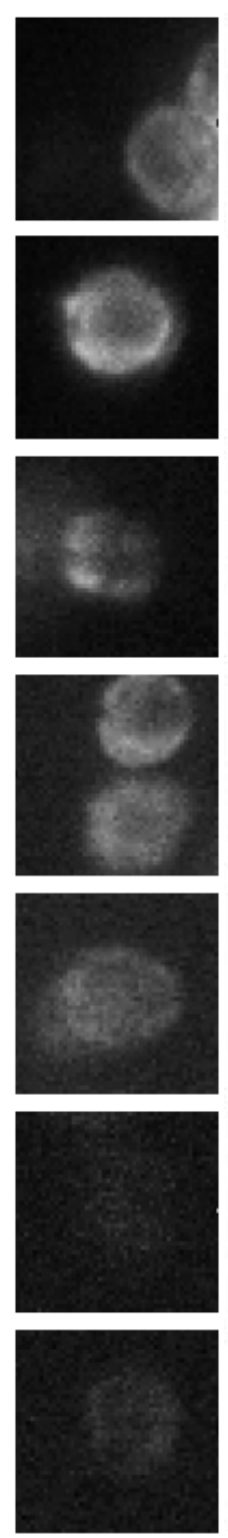

CD11C
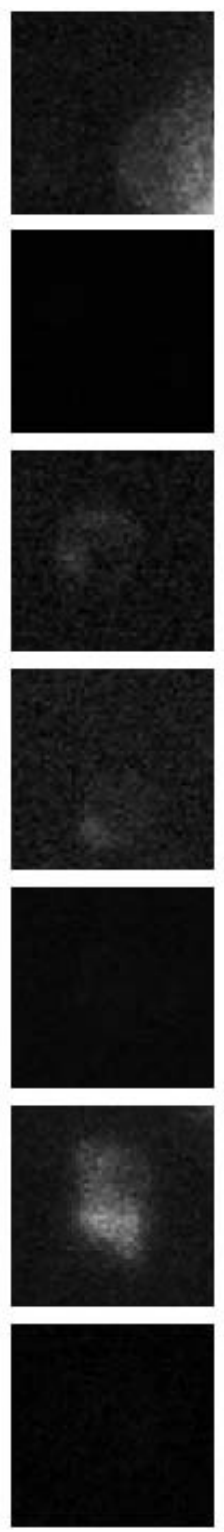

CoV-2 Spike RNA

Composite
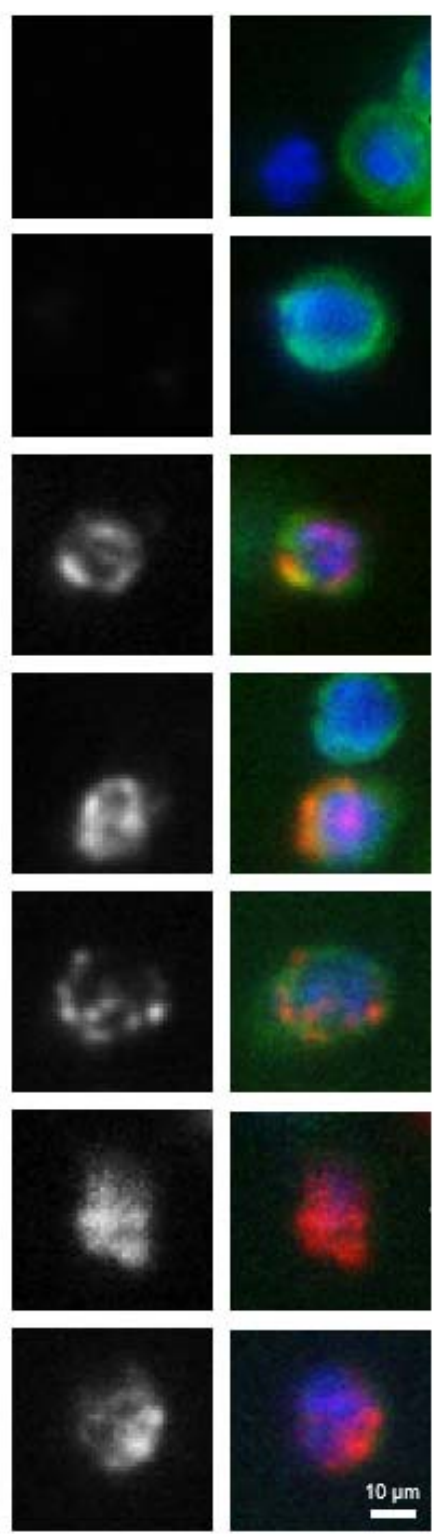

$10 \mu \mathrm{m}$

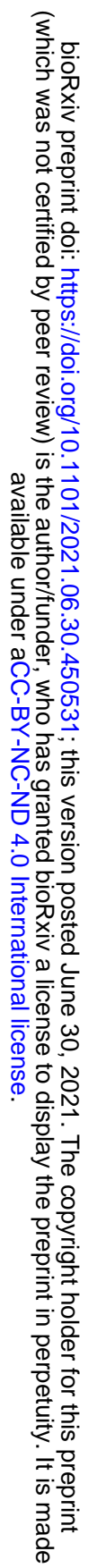

